\title{
Analysis of subclinical infections and biofilm formation in cases of capsular contracture after silicone augmentation rhinoplasty: Prevalence and microbiological study
}

\author{
Supasid Jirawatnotai, Bhakabhob Mahachitsattaya \\ Plastic and Reconstructive Surgery Unit, Lerdsin Hospital, Ministry of Public Health, Bangkok, Thailand
}

Background Implant-related deformities in aesthetic rhinoplasty are a major problem for rhinoplasty surgeons. Capsular contracture is believed to be the pathological cause of delayed contour deformities, comparable to breast implant-related contracture. This study investigated the prevalence of bacterial biofilms and other epidemiological factors related to capsular contracture in cases of silicone augmentation rhinoplasty.

Methods Thirty-three patients who underwent corrective rhinoplasty due to a delayed contour deformity or aesthetic revision after implant rhinoplasty were studied from December 2014 to December 2016. All recruited patients received surgical correction by the authors. The patients were categorized by clinical severity into four grades. Demographic data and related confounding factors were recorded. Samples of capsular tissue and silicone removed from each patient were analyzed for the presence of a biofilm by ultrasonication with bacterial culture and scanning electron microscopy.

Results Thirty-three paired samples of capsular tissue and silicone implants from the study group were analyzed. Biofilms were detected in one of 10 subjects (10\%) with grade 1 contracture, two of four (50\%) with grade 2 contracture, 10 of $14(71.40 \%)$ with grade 3 contracture, and four of five $(80 \%)$ with grade 4 contracture $(\mathrm{P}<0.05)$. The organisms found were Staphylococcus epidermidis (47.10\%), coagulase-negative staphylococci (35.30\%), and Staphylococcus aureus (17.60\%).

Conclusions As with breast implant-related capsular contracture, silicone nasal augmentation deformities likely result from bacterial biofilms. We demonstrated the prevalence of biofilms in patients with various degrees of contracture. Implant type and operative technique seemed to have only vague correlations with biofilm presence.

Keywords Rhinoplasty / Silicone / Biofilm

\section{Correspondence:}

Supasid Jirawatnotai

Plastic and Reconstructive Surgery Unit, Lerdsin Hospital, Ministry of Public Health, 190 Silom Road, Bangrak, Bangkok 10500, Thailand Tel: +66-2-353-9801

Fax: +66-2-353-9756

E-mail: add345@gmail.com

This article was presented at the International Aesthetic Conference of Chang Gung Memorial Hospital, on September 22-24, 2017, in Taipei, Thailand.

Received: 13 Jul 2018 • Revised: 30 Nov 2018 • Accepted: 4 Jan 2019

pISSN: 2234-6163・ elSSN: 2234-6171 • https://doi.org/10.5999/aps.2018.00864• Arch Plast Surg 2019;46:160-166

\section{INTRODUCTION}

In Southeast Asia, an under-projected tip and flat nasal dorsum are the most common unattractive features among patients seeking rhinoplasty. Silicone implants are the most common alloplastic material that has been used for nasal augmentation. Be- 
fore the era of hybrid rhinoplasty, in which an implant is combined with cartilage grafting, silicone implants were extensively popularized by cosmetic surgeons due to their biocompatibility, unlimited amount, and low price. Despite the increased awareness of the risks associated with silicone implants, including infection, malposition, calcification, and skin complications [1], many surgeons still perform augmentation rhinoplasty using silicone implants as their first choice of material. This has long been considered acceptable in Thailand and many Southeast Asian countries due to patients' naturally thicker skin, which allows mild thinning and contour changes to be better tolerated.

Most plastic surgeons are familiar with breast implant-related capsular contracture, which causes delayed contour changes in breasts. Capsule formation around silicone implants in breast surgery has been widely examined, and subclinical bacterial colonization (i.e., biofilm formation) was found to be an important factor provoking capsular contracture [2]. Surgeons practicing cosmetic surgery have been exploring new surgical techniques to provide safe and effective ways to improve implant augmentation rhinoplasty, including innovative shaping of implants, reducing implant stiffness, and changing the implant material. These methods have not proven to be highly effective, and implant-related deformities can still be seen. Such deformities are the most common reason for revision rhinoplasty performed by board-certified plastic surgeons in many countries.

In 1999, a Staphylococcus epidermidis biofilm was first detected in a patient with recurrent capsular contracture after silicone implantation, and this biofilm was argued to be a factor that accelerated periprosthetic capsule formation [3]. A biofilm is a congregation of microorganisms within the matrix of extracellular polymeric substance, which protects the microbes from therapeutic agents. Biofilms prompt chronic inflammation and progressive fibrosis, leading to contracture. In 2007, Jung et al. [4] reported that capsular tissue on the rim of a nasal silicone implant appeared to have similar histopathology to that of a sample from a silicone breast implant. Based on these observations, we designed a prospective study to further investigate the prevalence of bacterial biofilms in patients with delayed nasal contour deformity after silicone augmentation rhinoplasty.

\section{METHODS}

Thirty-three patients who underwent augmentation rhinoplasty using a silicone implant and required revision between December 2014 and December 2016 were included in the study (32 females and 1 male, 19-43 years of age). All recruited patients received surgical correction by the authors. The silicone implant-associated deformities included deviation, dorsal edge de- marcation, impending extrusion, and short nose deformity. All contour changes must have occurred at least 6 months after the last rhinoplasty procedure. Cases of immediate deviation or any deformity occurring within the first 6 months after surgery were excluded to minimize the likelihood that the deformity was caused by an error in the surgical technique. Written informed consent, including a photo release form for publication, was obtained from each patient. The study protocol was approved by Institutional Ethics Committee of Lerdsin Hospital (IRB approval number: ST3036/17/57).

Nasal contour deformities after silicone augmentation were classified into four grades according to severity, as described by Kim et al. [5]: grade 1, natural appearance (unsatisfactory aesthetic result); grade 2, unnatural lateral margin of implant; grade 3 , clearly identified implant deviation; and grade 4 , short nose deformity (Fig. 1). Patients who presented with a calcified capsule or any sign of infection (i.e., nasal erythema, pus discharge, current antibiotic use) or had received a filler injection were excluded from the study. Patients with a history of nasal bone injury or had received prostheses other than silicone were also excluded.

\section{Surgical technique}

The nasal hair was clipped and the surgical field was prepared with standard antiseptic solution (chlorhexidine gluconate in water), including inside the nostrils. In cases of mild contracture, the endonasal (closed) approach was used. The capsular tissue was identified and removed immediately after the incision was deepened from the infracartilagenous point to the capsule at the caudal end of the implant. The silicone implant was then retrieved and sent for processing. The cephalic half of the capsule was left in place to prevent soft tissue compromise, and the new implant was inserted into the newly created pocket underneath the existing pocket. For more severely deformed cases that warranted the open approach, an inverted-V transcolumellar incision was made and proceeded to the capsular tissue covering the implant. The sampled capsular tissue and implant were sent for processing before further dissection and reconstructive steps were performed (Fig. 2). Revision of augmentation was done by placing a new implant combined with cartilage grafting or total reconstruction with autologous rib cartilage. In severe cases, the thick and deformed capsule was totally removed and soft tissue coverage was reinforced with a fascial graft from the postauricular soft tissue or external oblique fascia from the chest.

\section{Sample collection}

Each capsule and its accompanying silicone implant were care- 


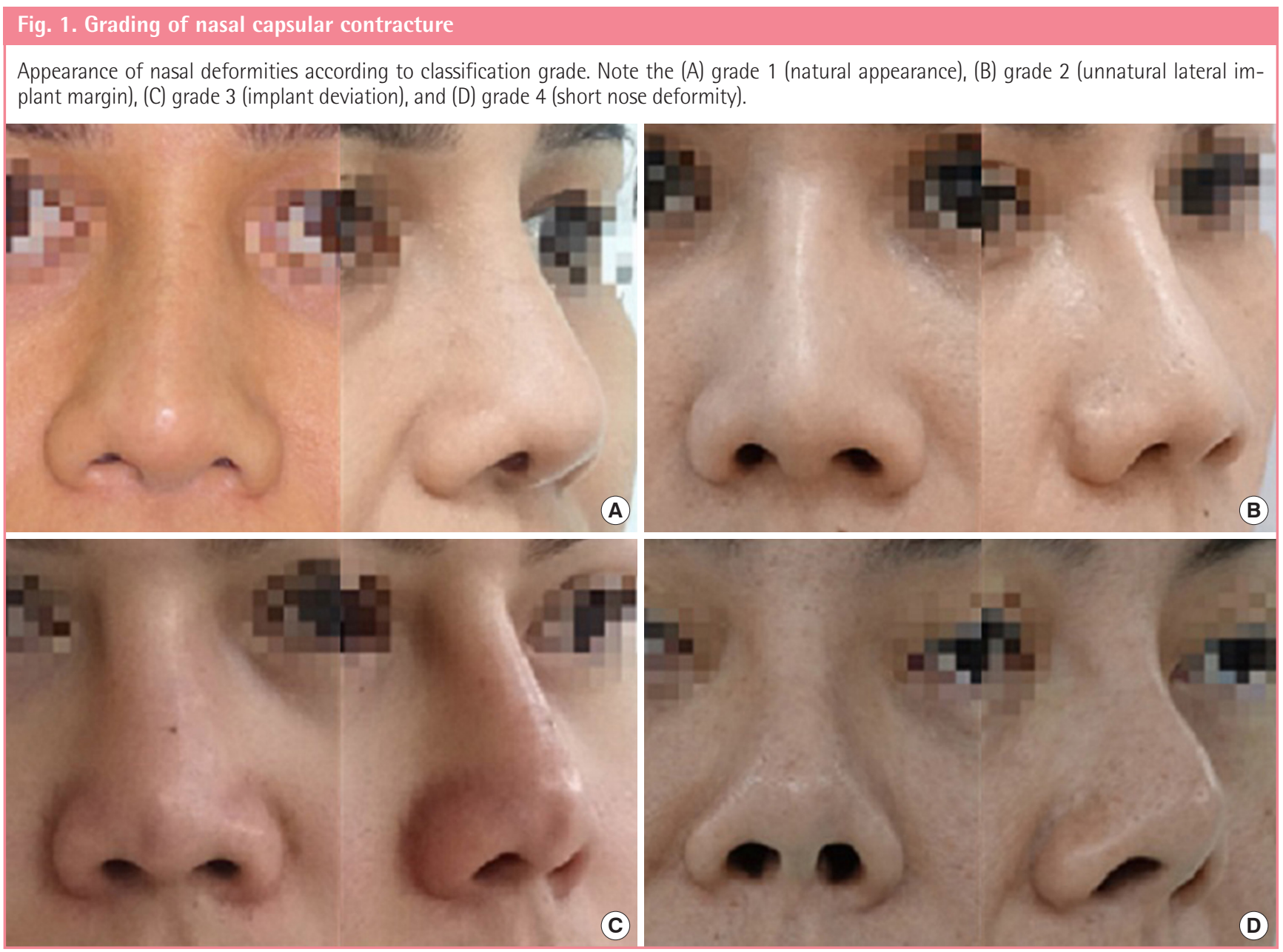

Fig. 2. Intraoperative findings

Intraoperative findings during a revision rhinoplasty procedure. (A) An implant was identified in situ. (B) Capsular tissue can be clearly identified after implant removal.
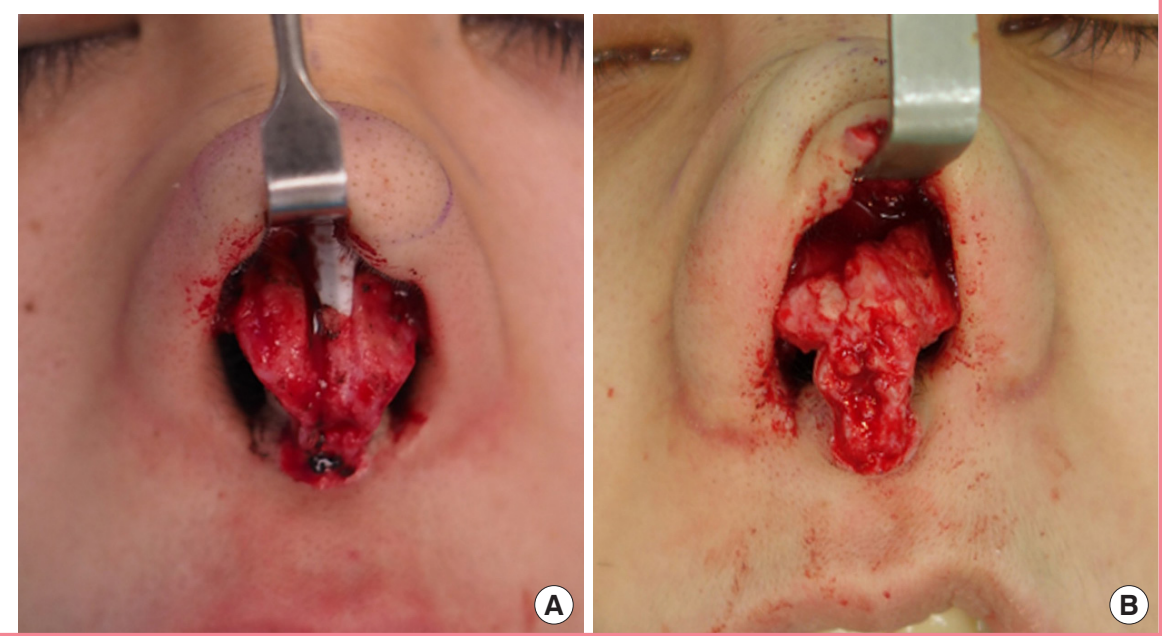

fully cut into small pieces, and two representative samples of the capsule and implant $\left(0.5 \times 0.5 \times 0.5 \mathrm{~cm}^{3}\right.$ each; one from the cephalic portion and the other from the caudal portion of the specimen) were selected for analysis by scanning electron microscopy (SEM) and bacterial culture. Each sample of the cap- sule and its associated silicone implant was individually placed into a sterile test tube with $15 \mathrm{~mL}$ of phosphate-buffered saline and sent for a microbiological examination. The other pair of capsule and silicone implant samples was fixed with $3 \%$ glutaraldehyde and $0.05 \%$ ruthenium red in phosphate for SEM. 


\section{Fig. 3. Scanning electron micrographs}

Examples of scanning electron microscopy findings of capsular tissue and implant surfaces. (A) The sectioned capsular tissue removed from a patient exhibiting capsular contracture demonstrated evidence of coccoid cells in a biofilm or thin fibrils $(\times 15,000)$. (B) The sectioned nasal silicone removed from a patient with capsular contracture showed coccobacilli cells in amorphous accumulations $(\times 6,500)$.
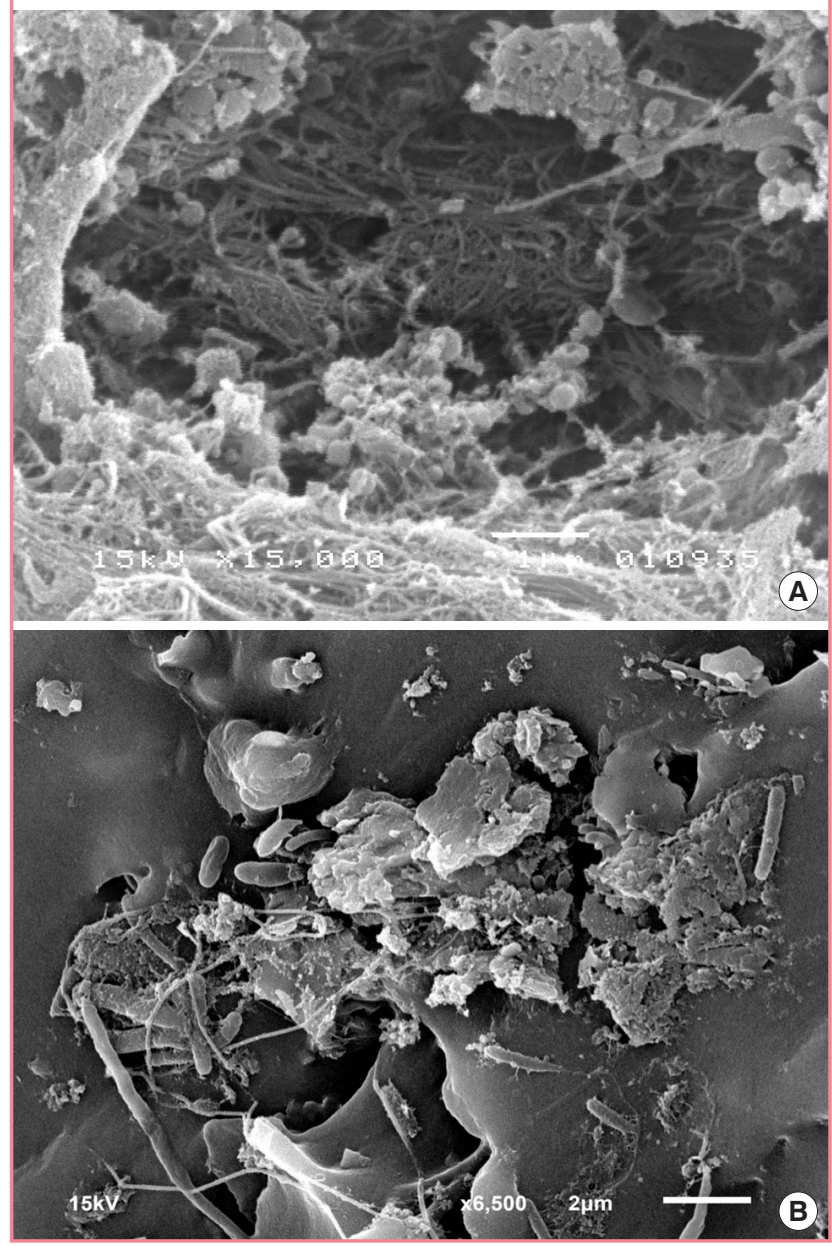

\section{Microbiological examination}

A portion of the capsule and silicone implant was washed twice in phosphate-buffered saline by vigorous shaking for 3 minutes. Ultrasonication of samples was performed at 50 to $60 \mathrm{~Hz}$ for 20 minutes. The prepared samples were scraped into a blood agar culture tray and set at room temperature for 1 day. The solution surrounding the samples was dropped into cooked meat medium and placed in an incubator at $37^{\circ} \mathrm{C}$ for 7 days. Microorganisms were then identified through microscopy.

\section{Scanning electron microscopy}

The specimens of each capsule and implant were washed with buffer and ruthenium three times, then dehydrated with increasing concentrations of alcohol. The dehydrated samples

\begin{tabular}{|c|c|c|c|c|}
\hline Characteristic & $\begin{array}{l}\text { Grade } 1 \\
(n=10)\end{array}$ & $\begin{array}{c}\text { Grade } 2 \\
(n=4)\end{array}$ & $\begin{array}{l}\text { Grade } 3 \\
(n=14)\end{array}$ & $\begin{array}{c}\text { Grade } 4 \\
(n=5)\end{array}$ \\
\hline Age (yr) & $30(19-41)$ & $27.5(23-36)$ & $30.14(20-43)$ & $33.8(24-43)$ \\
\hline \multicolumn{5}{|l|}{ Sex } \\
\hline Female & 10 & 4 & 13 & 5 \\
\hline Male & 0 & 0 & 1 & 0 \\
\hline $\begin{array}{l}\text { Duration from } \\
\text { surgery to } \\
\text { contracture (mon) }\end{array}$ & $21.7(10-48)$ & 29 (24-38) & $28.57(12-48)$ & $25.4(15-36)$ \\
\hline
\end{tabular}

were coated with gold by a sputter deposition machine. The coated samples were checked for groups of bacteria using an XL30 SEM (Philips Healthcare, Amsterdam, the Netherlands) at an accelerating voltage of $10 \mathrm{kV}$. Biofilms were identified by evidence of coccoid cells in lattices of thin fibrils or amorphous accumulations (Fig. 3).

\section{Analysis}

A sample was defined to be biofilm-positive if more than $10^{5}$ colony-forming units per milliliter were detected by culture and if SEM revealed bacterial colonization with a biofilm structure. If a specimen showed evidence of bacterial colonization on SEM but no growth on culture, it was considered biofilm-negative. If the bacterial culture was positive without colonization on SEM, the result was also considered negative. Evidence of bacteria in any specimen was assumed to be the result of contamination unless confirmed by both methods.

\section{Statistical analysis}

The prevalence of biofilms in patients with each grade of contracture was reported. Differences between groups were determined using chi-square analysis SPSS version 16 (SPSS Inc., Chicago, IL, USA). P-values less than 0.05 were considered to indicate statistical significance.

\section{RESULTS}

Thirty-three capsule and 33 implant samples were collected from 33 patients during the 24-month period. Thirty-two patients were female (97\%) and one was male (3\%). Their average age was 30.33 years (range, 19-43 years). The average time from implantation to symptomatic contracture was 26.06 months (range, 10-48 months) (Table 1).

The prevalence of biofilms increased with the severity of contracture, as biofilms were only found in $10 \%$ of patients with grade 1 contracture, but the prevalence increased to $50 \%, 71.4 \%$, and $80.0 \%$ of patients with grade 2,3 , and 4 contracture, respec- 
tively $(\mathrm{P}<0.05)$ (Fig. 4).

The most common organism identified in the biofilms by culture was Staphylococcus epidermidis (47.10\%), followed by coagulase-negative staphylococci (35.30\%) and Staphylococcus aureus (17.60\%).

The prevalence of biofilms was compared according to various

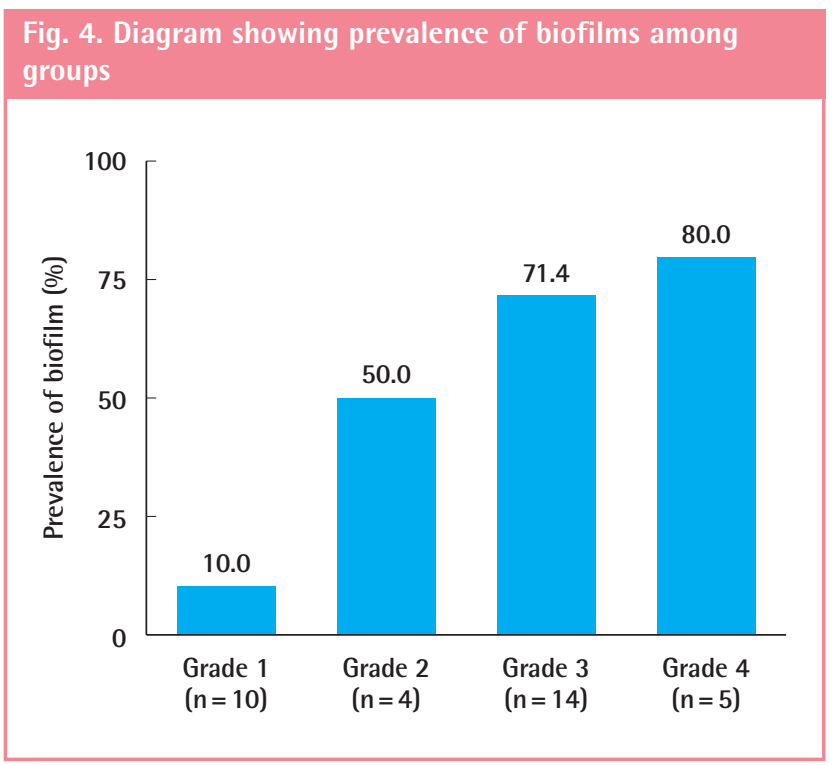

clinical conditions. No statistical significance was found in the prevalence of biofilms according to the institution where patients underwent rhinoplasty (hospital vs. private clinic), the shape of the implant (L-strut vs. non-strut), the plane of implant placement (subcutaneous vs. subperiosteal), or the type of silicone (pre-shaped vs. carved block) (Table 2).

\begin{tabular}{|c|c|c|}
\hline Condition & Biofilm-positive & P-value ${ }^{a)}$ \\
\hline Institution & & 0.247 \\
\hline Hospital & 6/13 (46.2) & \\
\hline Private clinic & $11 / 20(55.0)$ & \\
\hline Shape of implant & & 0.732 \\
\hline L-strut & $5 / 12(41.7)$ & \\
\hline Non-strut & 12/21 (57.1) & \\
\hline Plane of placement & & 0.437 \\
\hline Subcutaneous & 1/3 (33.3) & \\
\hline Subperiosteum & 16/30 (53.3) & \\
\hline Type of implant & & 0.510 \\
\hline Pre-shaped & $5 / 8(65.5)$ & \\
\hline Carved block & $12 / 25(48.0)$ & \\
\hline \multicolumn{3}{|c|}{$\begin{array}{l}\text { Values are presented as number/total number (\%). } \\
\text { a)The chi-square test was used to compare the prevalence of biofilms betwee } \\
\text { the subgroups of each clinical condition. }\end{array}$} \\
\hline
\end{tabular}

\section{Fig. 5. A case of grade 4 contracture}

Preoperative $(A-C)$ and 2-month postoperative images (D-F) of a patient with short nose deformity (grade 4 contracture) were demonstrated. Total capsulectomy, soft tissue release, dorsal augmentation with a new implant, and tip cartilage construction with an autogenous graft were performed.
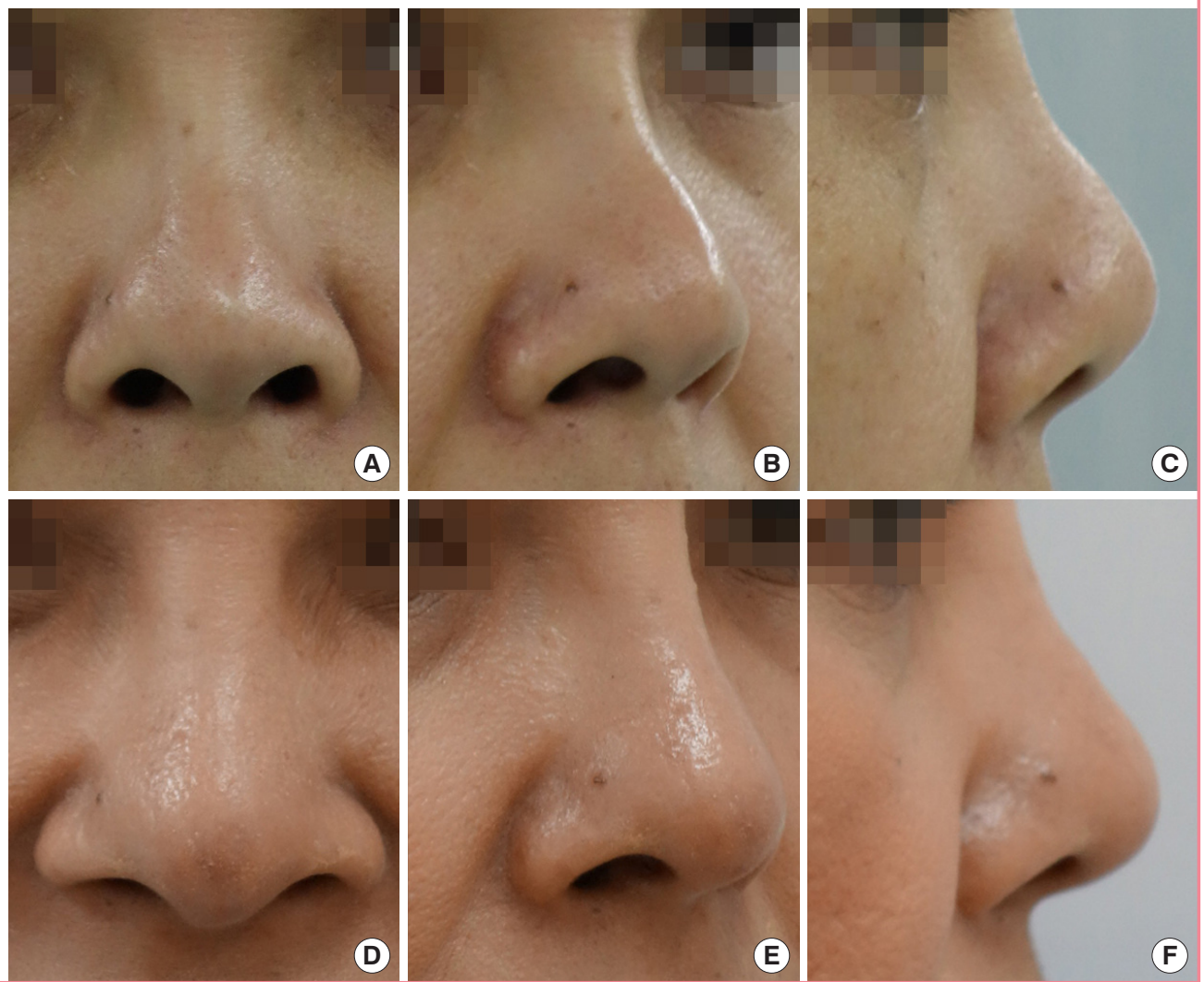

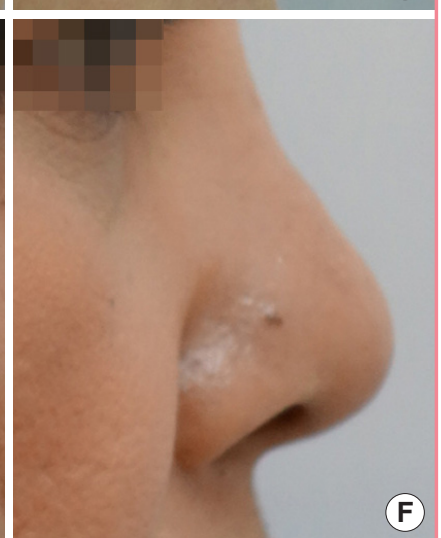


Clinical photographs of representative patient with grade 4 contracture are presented in Fig. 5.

\section{DISCUSSION}

Subclinical bacterial infections have long been demonstrated to be strongly related to biofilm formation. The amorphous substances and network of thin fibrils produced by pathogenic bacteria stimulate chronic inflammation, which results in endogenous capsular fibrosis and contracture [3]. This study was designed to investigate the prevalence of biofilms and their distribution among patients with various grades of nasal capsular contracture. Compared to surgical technique and the physical properties of the prosthesis, the importance of biofilm is dramatically underrated by many rhinoplasty surgeons. It is impossible to observe the presence of a biofilm by gross examination of capsular tissue during revision rhinoplasty; therefore, mechanical removal of the capsule with its associated implant may be the most effective measure to disrupt the biofilm and enable surface disinfection. Further reconstructive steps are needed if removal of capsular tissue weakens the soft tissue quality of the nose.

The nature of rhinoplasty procedures using silicone implantation makes silicone implants particularly susceptible to bacterial contamination and subsequent biofilm formation, due to the site of implant placement and surgical field contamination. Biofilms have been previously reported to develop starting in the second hour after other procedures [6]. Researchers demonstrated that inoculation of staphylococci, which are normal inhabitants of the nasal cavity, during implantation of silicone prostheses resulted in thick capsule formation, and there was a sequential relationship between the degree of contracture and number of bacteria $[7,8]$. A significant correlation between breast Baker capsular contracture grades 3 and 4 and biofilms has been reported [9]. We had demonstrated similar correlation in nose capsular contracture. Bacterial biofilms can also be found in other implantable devices, such as joint prostheses, penile prostheses, vascular grafts, Foley catheters, and contact lenses [10-14]. Adjustment of the nose shape necessitates implant manipulation throughout the procedure. Nasal implants might therefore be at a higher risk for contamination than other prostheses.

Biological approaches for identifying biofilms include semiquantitative staining, measurements of dried biomass, protein or DNA quantification, and assessments of residual viable organisms through standard microbial culture techniques. Each biological detection method has advantages and deficiencies, but they all provide only indirect measures of biofilm formation ability and are prone to operator-induced variability. In contrast, direct imaging of a biofilm provides information on its structural characteristics, its interactions with the surface, and its spatial distribution. SEM has been used extensively for qualitative observations of biofilm disruption due to its high resolution, and it is usually applied in combination with biological assays.

Previous researchers have described bacterial biofilms in other explanted alloplastic facial implants. Interestingly, silicone implants appeared to have less severe biofilms than porous polyethylene implants, which was suggested to be a result of their different surface textures [15]. SEM is an excellent tool to identify areas of biofilm formation, and we further investigated the causative pathogens by bacterial culture. We hypothesized that manipulation of the implant by carving or texture imprinting onto its surface might induce biofilm formation, but such an association was not shown in our results.

Recently, an association between breast implants and the development of anaplastic large-cell lymphoma (ALCL) has been observed. Samples from ALCLs showed high mean numbers of bacteria, as did non-tumor capsules; however, different species of bacteria were identified between both groups [16]. Our findings that staphylococci were present in a majority of samples are compatible with previous findings from non-tumor capsules in cases of breast capsular contracture.

Our results confirmed that biofilms are responsible for late contour deformities after silicone augmentation rhinoplasty, as well as in cases of breast implant-related contracture. Every effort should be made to minimize bacterial contamination and bleeding intraoperatively. Preoperative and postoperative antibiotic therapy is strongly recommended because the nasal cavity is readily contaminated by flora.

The degree of contracture after silicone augmentation rhinoplasty was classified according to the appearance of the nose at a long temporal interval after surgery. We demonstrated that the prevalence of biofilms increased along with the severity of the deformity. However, due to the small sample size, further large multi-center analyses are needed to clarify questions related to the risk and safety of biofilms in rhinoplasty and preventive measures that can be taken.

In conclusion, as in breast implant-related capsular contracture, silicone nasal augmentation deformities likely result from bacterial biofilms. We demonstrated the prevalence of biofilms in patients with various degrees of contracture. Further studies with a larger sample size are needed for a more objective analysis of causative risk factors for biofilm formation, as well as to obtain statistical significance. No conclusions regarding implant type or operative technique with respect to the risk of biofilm induction can be drawn from this initial analysis. 


\section{NOTES}

\section{Conflict of interest}

No potential conflict of interest relevant to this article was reported.

\section{Ethical approval}

The study was approved by the Institutional Review Board of Lerdsin Hospital (IRB No. ST3036/17/57) and performed in accordance with the principles of the Declaration of Helsinki. Written informed consents were obtained.

\section{Patient consent}

The patients provided written informed consent for the publication and the use of their images.

\section{Author contribution}

Idea and surgical procedure: Jirawatnotai S. Analysis and data collection: Mahachitsattaya B. All authors verified the analytical methods and discussed the results and contributed to the final manuscript.

\section{ORCID}

Supasid Jirawatnotai https://orcid.org/0000-0002-3831-473X Bhakabhob Mahachitsattaya https://orcid.org/0000-00026897-8764

\section{REFERENCES}

1. Hiraga Y. Complications of augmentation rhinoplasty in the Japanese. Ann Plast Surg 1980;4:495-9.

2. Baker JL Jr. Augmentation mammaplasty. In: Owsley JQJr, Peterson RA., editors. Symposium on aesthetic surgery of the breast. St. Louis: C.V. Mosby; 1978. p. 256-63.

3. Deva AK, Chang LC. Bacterial biofilm: a cause for accelerated capsular contracture? Aesthet Surg J 1999:19:130-3.

4. Jung DH, Kim BR, Choi JY, et al. Gross and pathologic analysis of long-term silicone implants inserted into the human body for augmentation rhinoplasty: 221 revision cases.
Plast Reconstr Surg 2007;120:1997-2003.

5. Kim YK, Shin S, Kang NH, et al. Contracted nose after silicone implantation: a new classification system and treatment algorithm. Arch Plast Surg 2017;44:59-64.

6. Dower R, Turner ML. Pilot study of timing of biofilm formation on closed suction wound drains. Plast Reconstr Surg 2012;130:1141-6.

7. Shah Z, Lehman JA Jr, Tan J. Does infection play a role in breast capsular contracture? Plast Reconstr Surg 1981;68: 34-42.

8. Tamboto H, Vickery K, Deva AK. Subclinical (biofilm) infection causes capsular contracture in a porcine model following augmentation mammaplasty. Plast Reconstr Surg 2010;126:835-42.

9. Pajkos A, Deva AK, Vickery K, et al. Detection of subclinical infection in significant breast implant capsules. Plast Reconstr Surg 2003;111:1605-11.

10. Tunney MM, Dunne N, Einarsson G, et al. Biofilm formation by bacteria isolated from retrieved failed prosthetic hip implants in an in vitro model of hip arthroplasty antibiotic prophylaxis. J Orthop Res 2007;25:2-10.

11. Vinh DC, Embil JM. Device-related infections: a review. J Long Term Eff Med Implants 2005;15:467-88.

12. Bergamini TM, Bandyk DF, Govostis D, et al. Infection of vascular prostheses caused by bacterial biofilms. J Vasc Surg 1988;7:21-30.

13. Rogers J, Norkett DI, Bracegirdle P, et al. Examination of biofilm formation and risk of infection associated with the use of urinary catheters with leg bags. J Hosp Infect 1996; 32:105-15.

14. Tran PL, Huynh E, Pham P, et al. Organoselenium polymer inhibits biofilm formation in polypropylene contact lens case material. Eye Contact Lens 2017;43:110-5.

15. Walker TJ, Toriumi DM. Analysis of facial implants for bacterial biofilm formation using scanning electron microscopy. JAMA Facial Plast Surg 2016;18:299-304.

16. Hu H, Johani K, Almatroudi A, et al. Bacterial biofilm infection detected in breast implant-associated anaplastic largecell lymphoma. Plast Reconstr Surg 2016;137:1659-69. 